\section{RF20 PRIMARY HEALTH CARE PROFESSIONALS VIEWS OF REMINDERS IN ELECTRONIC PATIENT RECORDS}

EV Cecil ${ }^{*}$, L Dewa, R Ma, A Majeed, P Aylin. Primary Care and Public Health, Imperial College London, London, UK

\subsection{6/jech-2019-SSMabstracts.135}

Background Primary care administrative systems contain the medical records of patients' contacts with primary care services. These data are used to create electronic reminders at the point of care. The reminders, or pop-up prompts, support clinicians to provide good quality care, safe prescribing and to save money. It is suggested that the increasing use of reminders has led to desensitisation and a tendency to ignore the information provided. Our study aimed to explore primary healthcare professionals' (PHCP) attitudes towards electronic reminders in primary care, to support their enhancement.

Methods Fifteen semi-structured interviews were conducted with general and nurse practitioners based in North West London and Yorkshire, England. PHCPs were selected as a national representative sample across various metrics (position or experience of the PHCP; factors relating to the practice; and the demographic characteristics of the practice population). Topic areas included i) experiences of reminders; ii) perceptions of the validity of the information provided; iii) reminder-related behaviours and iv) views on improvement. We analysed data using a framework approach.

Results We found that PHCPs were familiar with the reminders in their clinical systems. PHCPs believed most were important and supported their work by providing up to date, evidence based information. Significant barriers towards reminders were time constraints; numbers; and the fact that reminders interrupted their natural patient interaction. PHCPs felt frustrated that data requested by a reminder and collected in contacts with other healthcare providers was not shared (for example a blood pressure reading or smoking status). Poorly designed or oversensitive reminders were considered particularly annoying. Feelings towards reminders were not consistent across the participants and often related to experience, roles, consultation styles and interests. Few PHCPs had experienced or heard of negative outcomes after ignoring the information in a reminder, but some did feel guilty or concerned that ignoring a reminder would reflect badly on them. PHCPs felt more training was needed to efficiently manage reminders but highlighted that managing reminders was also the responsibility of the patients.

Conclusion Improving reminders must ensure integration between health care administrative systems (primary, community and hospital); develop more refined algorithms; and consider cognitive workflow of PHCPs.

\section{RF21 WHY DO SOME GPS AND PRACTICE NURSES IN THE UK CONTINUE TO PRESCRIBE ANTIBIOTICS INAPPROPRIATELY? A QUALITATIVE ANALYSIS OF HEALTH PROFESSIONALS' ANTIBIOTIC PRESCRIBING IN PRIMARY CARE IN THE NHS}

RE Glover*, A Fraser. Health Services Research and Policy, London School of Hygiene and Tropical Medicine, London, UK

10.1136/jech-2019-SSMabstracts. 136

Background Antibiotic prescribing in primary care has decreased over the last five years. Nevertheless, this remains an area of concern as antibiotic resistance rates continue to increase. Some prescribers continue to prescribe inappropriately - i.e. in contradiction of clinical guidelines. This qualitative study undertakes thematic analysis to determine the attitudes and perceptions of these professionals about inappropriate prescribing.

Methods We draw on data from our evaluation of the UK's five-year antimicrobial resistance strategy, undertaken from 2015-18 funded by the Department of Health and Social Care. We conducted 73 semi-structured interviews across six case study sites at the CCG level or equivalent in each of the four nations in the UK. Relevant informants in each trust were theoretically sampled in order to capture a mix of professionals in each case study site (including GPs, nurse prescribers, antimicrobial pharmacists, medicines management trust professionals, microbiologists, hospital doctors with opinions on primary care, and commissioners with oversight roles). Analysis was undertaken drawing on inductive and deductive logics.

Results In primary care, antibiotics have a symbolic potency that is constructed and mediated through the interactions of the prescriber and the patient. These interactions produce a negotiated understanding between both parties in relation to the significance and symbolism of an antibiotic prescription. Our analysis highlights how decisions to prescribe an antibiotic may be influenced by the context of competing pressures extrinsic to the patient-provider relationship, including time, risk, and responsibility. In certain circumstances this may lead to the inappropriate prescription of an antibiotic script.

Influenced by the theory of negotiated order, ${ }^{1}$ we explore how different approaches towards antibiotic-seeking behaviour by patients are interpreted by prescribers. We highlight how extrinsic factors may influence co-produced care, and consequently impact upon a patient or provider's agency, including: (1) rapid diagnostics, which aim to reduce uncertainty in a consultation; and (2) disruptions to medical hierarchies, such as attaching an antimicrobial pharmacist to a GP practice in order to monitor the appropriateness of antibiotic prescriptions.

Conclusion How providers negotiate their patients' antibioticseeking behaviour is linked to temporal factors, professional experience, perceptions of risk, and culturally mediated understandings of 'appropriateness'. Future efforts to reduce antibiotic prescribing in community settings may be achievable by pulling on extrinsic levers, rather than sacrificing the patientprovider relationship.

\section{REFERENCE}

1. Strauss A, Schatzman L, Ehrlich D, Bucher R, Sabshin M. (1963). The hospital and its negotiated order. In Friedson E. (ed). The Hospital in Modern Society. Free Press, Glencoe, NY, 147-169.

\section{RF22 THE ROLE OF POWER IN REGULATING ONLINE ADVERTISING OF HIGH IN FAT, SUGAR AND SALT FOOD AND BEVERAGES TO CHILDREN: PARENT AND STAKEHOLDER PERSPECTIVES}

LE White*, S Chambers, K Skivington, S Hilton. MRC/CSO Social and Public Health Sciences Unit, University of Glasgow, Glasgow, UK

10.1136/jech-2019-SSMabstracts. 137

Background In the United Kingdom (UK) increasing childhood obesity rates may be indicative of a lack of regulation of 
corporate influences contributing to an obesogenic environment. A current focus in childhood obesity policy debates is the role that online advertising of high in fat, sugar and salt (HFSS) food and beverages plays as a driver of childhood obesity and what regulation is required. However, there is a lack of research in this area as to public acceptability of regulation of online advertising of HFSS products as a viable policy solution. This study examines the perceptions of parents and stakeholders in regulating this online environment to answer how the regulation of online advertising of HFSS products to children is viewed in the UK?

Methods Three qualitative methods were triangulated: 1) eight focus groups with parents who have children aged between five and 15 years old; 2) scoping review of 85 responses to a 2016 Committees of Advertising Practice consultation on nonbroadcast advertising to children; and 3) 11 stakeholder interviews (industry, civil society, academics and government body). Data were analysed inductively and thematically using NVivo. Results Parents reported finding it increasingly difficult to attain a healthy diet for their children, competing with a multitude of pressures, including online advertising of HFSS products. Non-industry stakeholders agreed with this perspective, whereas industry stakeholders were sceptical as to the influence online advertising had in contributing to an obesogenic environment. In terms of attitudes to regulation, two views emerged from the three data sets: 1) support for increased regulation of online advertising of HFSS products, or 2) the continuation of the current self-regulatory model. Underpinning their views were concerns as to the distribution of power within the obesogenic environment, with the majority of parents and all non-industry stakeholders describing the food and beverage industry as possessing too much power, and government and parents possessing too little. In contrast, the remaining parents and industry stakeholders argued that government possessed too much power and as such infringed on individual autonomy.

Conclusion Parents' and stakeholders' views in this study largely aligned with Beauchamp's (1976) theory on social justice versus market justice. Although not generalisable, this study offers insights into how their perspectives on the distribution of power within the obesogenic environment may have informed their views on implementing increased regulation of the online advertising environment as a viable policy solution to tackle childhood obesity.

\section{RF23 'US' AND 'THEM': IDENTIFYING THE MOST SUITABLE APPROACH TO INVOLVING PATIENTS AND HEALTHCARE PROFESSIONALS IN A CONSENSUS PROCESS TO INFORM INTERVENTION DEVELOPMENT}

E Racine*, F Riordan, E Phillip, S McHugh, P Kearney. School of Public Health, University College Cork, Cork, Ireland

\subsection{6/jech-2019-SSMabstracts. 138}

Background Intervention development is a critical first step when conducting trials or observational studies. Research suggests that involving multiple stakeholders in this process increases the likelihood of developing interventions that are acceptable, engaging, feasible and effective. However, there is little guidance for researchers on the best ways to involve multiple stakeholders in a meaningful way. The aim of this Study Within A Trial (SWAT) is to identify the most suitable approach to involve patients and healthcare professionals in a consensus process to inform the development of the Improve Diabetes Eye-screening Attendance (IDEA's) intervention.

Methods This is a qualitative study. Three meetings were held to establish consensus on the content and delivery of the intervention. Meeting included 1 patients only, meeting 2 included a combination of patients and healthcare professionals and meeting 3 included healthcare professionals only. Stakeholders were asked to agree on intervention components which target patients and general practices. Each meeting was audio recorded and field notes were taken. After the meeting, semi-structured telephone interviews were carried to explore stakeholders' experiences of taking part. Data were transcribed verbatim and managed using NVivo V12 software. Thematic analysis was performed to identify themes relating to members' experiences of taking part.

Results All three meetings put forward feasible ideas which were incorporated into the final intervention. Each meeting had ideas which were not put forward by the other groups but were incorporated into the final intervention (Meeting $1=6$ ideas, Meeting 2=2 ideas and Meeting $3=5$ ideas). Thematic analysis suggests that stakeholders in meetings 1 and 3 felt comfortable expressing their opinions and grateful that they were given the opportunity to be heard. Stakeholders in meeting 2 felt they had to bold back on their opinions as they were aware that the other stakeholder group was in the room. They also felt that their contributions were undervalued by the other stakeholder group.

Conclusion Involving patients and healthcare professionals together in a consensus process is not a suitable approach to involvement. This study will guide researchers on the most suitable approach to involve patients and healthcare professionals in a consensus process and will contribute to the evolving literature on the potential impact of involving multiple stakeholders in the intervention development process.

\section{RF24 A SYSTEMATIC REVIEW AND META-ANALYSIS OF SCHOOL-BASED EDUCATIONAL INTERVENTIONS TO IMPROVE BODY COMPOSITION IN ADOLESCENTS}

${ }^{1} \mathrm{CM}$ Jacob, ${ }^{2,3} \mathrm{PL}$ Langdon, ${ }^{2,4} \mathrm{HM}$ Inskip, ${ }^{2} \mathrm{~T}$ Morris, ${ }^{2} \mathrm{C}$ Parsons, ${ }^{1,4} \mathrm{M}$ Hanson, ${ }^{1,3} \mathrm{~K}$ WoodsTownsend, ${ }^{2,4} \mathrm{~J}$ Baird*. Institute of Developmental Sciences, Faculty of Medicine, University of Southampton, Southampton, UK; ${ }^{2}$ MRC Lifecourse Epidemiology Unit, University of Southampton, Southampton, UK; ${ }^{3}$ Southampton Education School, Faculty of Social Sciences, University of Southampton, Southampton, UK; ${ }^{4}$ NIHR Southampton Biomedical Research Centre, University Hospital Southampton NHS Foundation Trust, Southampton, UK

\subsection{6/jech-2019-SSMabstracts. 139}

Background Adolescence is a period marked by critical changes in behaviours and body composition that place adolescents at an increased risk of becoming overweight and obese. Health education in schools has the potential to improve health behaviours by encouraging critical thinking about these issues. To develop sustainable interventions to prevent obesity, it is necessary to understand whether educational interventions during adolescence are effective, and which intervention elements are associated with improvements in BMI.

We addressed the question: do school-based educational interventions improve BMI in adolescence, and what intervention features are associated with effectiveness?

Methods We carried out a systematic review and meta-analysis of published intervention studies. We searched MEDLINE, PsycINFO, CINAHL, and ERIC from 2006 to 2017. Two 